\title{
Effects of lesions of the nucleus accumbens on food intake and body weight
}

\author{
MYRA O. SMITH \\ Colgate University, Hamilton, New York 13346 \\ and \\ ROBERT C. HOLLAND \\ Department of Anatomy, Faculty of Science, Mahidol University, Bangkok, Thailand
}

\begin{abstract}
Food and water intake and body weight of rats with lesions in the nucleus accumbens (NA) were monitored for several months, extending from a 4-week period prior to the lesion, through a 4-week postoperative period, gestation, parturition, to the end of a 3-week postpartum period. The most pronounced lesion-precipitated change related to energy balance was elevated consumption of food, relative to the experimental rats' own prelesion intake and to that of a sham control group. The apparent hyperphagia was not accompanied by increases in body weight. Indeed, except for prelesion weights, weights of rats with $50 \%$ or greater bilateral destruction of the NA or damage to at least $75 \%$ of the NA of one hemisphere lagged consistently, although not significantly (except during the postpartum period), behind those of animals with less extensive lesions and sham controls. Observations made during gestation and the postpartum period clearly indicated that lesions did not affect ability to respond to changes in the animal's physiology with appropriate adjustment of food intake. It was concluded that enhanced ingestion of food during the postlesion period was a secondary outcome of the increased demands imposed upon energy reserves by the hyperactivity and emotionality induced by the lesion.
\end{abstract}

In the course of conducting a pilot study on the effects of lesions in the nucleus accumbens (NA) on lactation and maternal behavior, it was noted that food, and occasionally water, intake was greatly elevated over prelesion levels. Consequently, the authors decided that, in the full-fledged study (Smith \& Holland, 1975), food and water consumption, along with body weight, should be recorded during an extended postlesion period.

As the present investigation was being launched, Lorens, Sorensen, and Harvey (1970) reported that lesions in the NA in male rats produced a transient increase in food intake, but no changes in water ingestion. Our study departed from that of Lorens et al. in that it used female rats and included observations of food and water intake during gestation and an ensuing 3-week postpartum period as well as during a postoperative premating period

This research was supported in part by a grant from the Rockefeller Foundation to the Faculty of Science, Mahidol University, and by funds provided by the Sloan Foundation to the Psychology Department, Colgate University. M. O. Smith was a postdoctoral Research Fellow, National Institute of Child Health and Development, N.I.H., Mahidol University, and a Postdoctoral Fellow, Sloan Foundation, Colgate University. She is currently Assistant Professor of Psychology at Colgate University. Send reprint requests to Myra O. Smith, Department of Psychology, Colgate University, Hamilton, New York 13346. R. C. Holland was a staff member of the Rockefeller Foundation, Visiting Professor of Anatomy at the Faculty of Science, Mahidol University. He is now chairman of the Department of Anatomy, Medical Education Program, Morehouse College, Atlanta, Georgia 30314. of a month. Moreover, records of body weight were kept throughout the duration of the project.

We were interested in determining not only whether a lesion in the NA would alter food and water consumption, but also whether this would be revealed in body weight, as it is, for example, in animals with damage to the ventromedial hypothalamus (VMH). The nature of the observed relationship between consumption and body weight, we believed, should suggest whether any increase in intake was a primary or secondary outcome of the lesion and help to clarify what role, if any, the NA has in the regulation of energy balance.

\section{METHOD}

\section{Subjects}

Subjects were 34 albino rats (Fisher strain) which, at the outset of the period of observation, were virgins weighing 130-150 g. They were fed an ad-lib diet of water and standard commercial food. They were individually housed in metal grid cages until 3 days before the anticipated date of parturition, when they were transferred to round plastic tubs, 12 in. in diameter and 5 in. in height, over which were fitted wire mesh lids.

\footnotetext{
Measurement of Food and Water Intake and Body Weight

Food intake was measured at the same hour daily by weighing containers, contents, and crumbs collected in boxes under the food bins and subtracting the total weight from the previous day's values. Contents were completely changed every 3 days. Water was similarly measured by weighing the bottles and contents and subtracting the total weight from measurements obtained the previous day. Bottles were washed and filled daily.
} 
When rats were transferred to the plastic tubs, food was placed in statle, wide-mouthed jars which enabled rats to nibble without spilling crumbs.

Rats were weighed daily at the same time of day on a small animal balance, measurements being made to the nearest tenth of a gram.

\begin{abstract}
Surgery
When rats approached $200 \mathrm{~g}$ in body weight, they were randomly assigned to the experimental group (NA-X) and to the sham control group (NA-SC). The former incurred bilateral electrolytic lesions in the NA at points corresponding to König and Klippel's (1963) stereotaxic coordinates A 8.9, L 1.0, H -0.8 and A 9.8, $\mathrm{L} 0.8, \mathrm{H}-0.5$. Twenty-four out of 28 animals survived lesioning.

Group NA-SC consisted of 10 rats that had been subjected to the same anesthetizing and surgical procedures as the NA-X group, with the exception that no current was passed through the electrode following its insertion into the NA.
\end{abstract}

\section{Procedure}

Observations of food and water intake and body weight which had been made for at least 4 weeks prior to surgery were continued for another 4- to 6-week period. The subjects were then mated with males introduced into their cages on the day of proestrus. Vaginal smears were checked for sperm the following day, and their presence marked Day 1 of gestation. In the absence of sperm for 2 consecutive days, the male was removed and replaced by a new one two cycles later. Food and water consumption and body weight were monitored throughout the 21-day gestation period and subsequently during the postpartum period for 21 days.

\section{Brain Reconstruction}

Reconstruction of lesion damage proceeded by matching projections of fuchsin-stained frontal sections of the brain with corresponding sections in the atlas of König and Klippel (1963). NA-X rats were first segregated into two groups on the basis of whether or not they showed an increase in water intake. Lesions which most characteristically distinguished the two groups were then sought. This procedure was repeated for determination of lesion sites most frequently associated with increased food consumption.

\section{RESULTS}

\section{Histology}

Lesions were large and often asymmetrical in shape and placement. In addition to damage to the NA, surrounding tissue was sometimes invaded, including the lateral and medial septum, diagonal band of Broca, and the caudate nucleus. Within the NA itself, size, shape, and site of lesions varied, as might be- expected of attempts to destroy a structure as large as this nucleus.

\section{Effects of NA Damage on Water Intake}

Postsurgical increase in water ingestion was observed in some subjects in both NA-X and NA-SC groups $(38 \%$ vs. $30 \%)$. In most of these instances, the effects were transient, lasting for no more than 1 week. In cases in which higher than normal quantities of water were consumed over a period of 2 or more weeks (three NA-X cases), degree of encroachment onto the septal area rather than extent of damage to the NA appeared to be the distinguishing variable.

\section{Effects of NA Damage on Food Intake and \\ Body Weight}

Postlesion period. More permanent and pronounced than changes in water intake levels was the enhancement of food consumption in the postoperative period. Food intake was significantly greater for NA-X animals, even at the end of a monthlong period. Moreover, this pattern was most consistently related to damage to the dorsomedial aspect of the NA between König and Klippel's anterior coordinates of A $8920 \mu$ and A $8620 \mu$. The degree of destruction of this area varied and formed the basis for the initial division of experimental animals into the subcategories described in Table 1. The statistical analyses summarized in Table 2 shows clearly the effect of the degree of involvement of this area upon food intake level.

Despite elevated food consumption, body weights of rats with destruction of at least $75 \%$ of the NA of one cerebral hemisphere or $50 \%$ or greater bilateral damage tended to lag consistently, although not statistically significantly, behind those of NA-SC rats (Table 3 ). There appeared to be an inverse relationship between food intake and body weight, the former being greatest when body weight was least (in the immediate postoperative period) and leveling off as the latter approached control values.

Gestation. During pregnancy, both lesioned and sham control rats displayed increased body weight.

Table 1

Subgrouping of Experimental Rats According to Size of Lesion in Dorsomedial One-Third of Accumbens

\begin{tabular}{llr}
\hline Category & \multicolumn{1}{c}{ Lesion Size } & N \\
\hline NA-X & $>50 \%$ bilateral & 9 \\
NA-X & $>75 \%$ in one NA and $<50 \%$ in the other & 5 \\
NA-X $_{3}$ & $50-75 \%$ in one NA and $<50 \%$ in the other & 6 \\
NA-X $_{4}$ & $<50 \%$ bilateral & 4 \\
Total & & 24 \\
\hline
\end{tabular}

Table 2

Effects of Lesion on Food Intake Average Daily Intake (Grams)

\begin{tabular}{lccccc}
\hline Group & $\mathrm{N}$ & $\begin{array}{c}\text { Pre- } \\
\text { lesion* }\end{array}$ & $\begin{array}{c}\text { Post- } \\
\text { lesion* }\end{array}$ & $\begin{array}{c}\text { Ges- } \\
\text { tation** }\end{array}$ & $\begin{array}{c}\text { Post- } \\
\text { partum*** }\end{array}$ \\
\hline $\mathrm{NA}_{1}+\mathrm{NA}_{2}$ & 14 & 13.67 & 19.73 & 20.15 & 18.18 \\
$\mathrm{NA}_{3}+\mathrm{NA}_{4}$ & 10 & 14.70 & 17.48 & 16.47 & 28.74 \\
$\mathrm{NA}-\mathrm{SC}$ & 10 & 13.86 & 15.83 & 17.16 & 29.91 \\
Analysis of Variance & & & & \\
$\mathrm{ms}_{\mathrm{b}}, \mathrm{df}=2$ & 3.27 & 45.94 & 46.99 & 514.33 \\
$\mathrm{~ms}_{\mathrm{w}}, \mathrm{df}=31$ & 13.20 & 9.09 & 10.38 & 20.32 \\
$\mathrm{~F}$ & .24 & 5.05 & 4.52 & 25.31 \\
$\mathrm{p}(\mathrm{F})$ & $>.05$ & $<.05$ & $<.05$ & $<.01$ \\
$\mathrm{NA}$ & & & & \\
$\mathrm{t}, \mathrm{df}=22$ & & 2.91 & 2.30 & 7.42 \\
$\mathrm{p}(\mathrm{t})$ & & $<.01$ & $<.05$ & $<.01$ \\
\hline
\end{tabular}

*Average over 28-day periods immediately prior to and following lesion.

**Average over Days 2-21 of gestation.

***Average over Days 1-21 postpartum. 
Table 3

Effects of Lesion on Body Weight (Grams)

\begin{tabular}{|c|c|c|c|c|c|c|}
\hline Group & $\mathrm{N}$ & Prelesion & Postlesion & Gestation & $\begin{array}{c}\text { Postpartum } \\
\text { Day } 1\end{array}$ & $\begin{array}{c}\text { Postpartum } \\
\text { Day } 21 \\
\end{array}$ \\
\hline $\begin{array}{ll}\mathrm{NA}_{1}+\mathrm{NA}_{2} & 1 \\
\mathrm{NA} & +\mathrm{NA}_{4} \\
\mathrm{NA}-\mathrm{SC} & 1\end{array}$ & $\begin{array}{l}14 \\
10 \\
10\end{array}$ & $\begin{array}{l}205.3 \\
204.8 \\
203.6\end{array}$ & $\begin{array}{l}214.0 \\
222.0 \\
224.0\end{array}$ & $\begin{array}{l}299.4 \\
310.2 \\
312.4\end{array}$ & $\begin{array}{l}224.2 \\
236.9 \\
233.8\end{array}$ & $\begin{array}{l}228.1 \\
244.0 \\
251.6\end{array}$ \\
\hline $\begin{array}{l}\text { Analysis of Va } \\
\mathrm{ms}_{\mathrm{b}}, \mathrm{df}=2 \\
\mathrm{~ms}_{\mathrm{w}}, \mathrm{df}=31 \\
\mathrm{~F} \\
\mathrm{p}(\mathrm{F})\end{array}$ & $\begin{array}{l}\text { arian } \\
1\end{array}$ & $\begin{array}{r}9.31 \\
51.02 \\
.18 \\
>.05\end{array}$ & $\begin{array}{r}332.33 \\
160.88 \\
2.06 \\
>.05\end{array}$ & $\begin{array}{r}579.81 \\
352.84 \\
1.69 \\
>.05\end{array}$ & $\begin{array}{r}536.20 \\
277.15 \\
1.93 \\
>.05\end{array}$ & $\begin{array}{r}1,759.66 \\
262.25 \\
6.67 \\
<.01\end{array}$ \\
\hline $\begin{array}{l}\mathrm{NA}_{1}+\mathrm{NA}_{2} \mathrm{vs} \\
\mathrm{t} \\
\mathrm{p}(\mathrm{t})\end{array}$ & vs. NA & & & & & $\begin{array}{l}4.01 \\
<.01\end{array}$ \\
\hline
\end{tabular}

Animals in Groups $\mathrm{NA}_{1}$ and $\mathrm{NA}_{2}$, in particular, continued to eat more than controls; yet their peak mean weight during pregnancy (day of parturition) and immediate postpartum weight did not deviate significantly from those of control rats. It is interesting to note that at birth their pups weighed significantly less than those of other dams [5.46 vs. $6.15 \mathrm{~g}$, $\mathrm{p}(\mathrm{t})<.05]$. There were no significant differences between groups in size of litter, abortions, and resorptions.

Postpartum period. For the first time since surgery, in the postpartum period control animals ate more as well as weighed more on the average than $\mathrm{NA}_{1}$ and $\mathrm{NA}_{2}$ dams. In all instances, when dams did not increase postpartum food consumption, they did not appear to be lactating. This was true of $92 \%$ of $\mathrm{NA}_{1}$ and $\mathrm{NA}_{2}, 40 \%$ of $\mathrm{NA}_{3}$ and $\mathrm{NA}_{4}$, and $20 \%$ of NA-SC animals.

\section{DISCUSSION}

That water intake is not associated with damage to the NA confirms the observation of Lorens et al. (1970). The result of our own attempts to correlate the occasional incidence of enhanced water ingestion with brain structure and those of Lorens and earlier workers (e.g., Harvey \& Hunt, 1965; Lubar et al., 1969) implicate the adjacent septal region.

Our finding of heightened food intake in NA-X rats is also consistent with the findings of Lorens et al. (1970). By demonstrating that increase in body weight did not accompany the increased food consumption, the present study suggests that we are not observing hyperphagia due to reduced sensitivity to satiety signals as appears to be the case in the classical ventromedial hypothalamus lesioned (VMH-X) rat. Indeed, the mechanism regulating food intake met very well the test of its efficiency in NA-X rats. On the basis of the following observations, it seems evident that rats were eating larger than normal quantities in order to attain and maintain an optimal body weight: (1) food intake was greatest in the immediate postoperative period, when body weight had dropped to its lowest level, and gradually decreased to near normal levels as body weight approached values characteristic of littermates in the NA-SC group; (2) the new nutrient demands of gestation were similarly reflected in the food intake levels of lesioned and control animals; and (3) in the postpartum periods those lesioned animals that failed to rear viable litters adjusted their eating behaviors to that of prepregnancy levels. In the last instance, body weight also returned to pregestational values.

Thus the NA-X rat, unlike the VMH-X counterpart, is not hyperphagic in the sense that it is eating beyond nutrient requirements (see, e.g., Anand, 1965; Hoebel, 1971). It is compelled to eat more in order to counterbalance the increased demands being imposed upon nutrient stores. There are two likely causes of the rapid and sustained depletion of energy stores: (1) increased gut motility leading to reduced assimilation of nutrients and (2) increased energy expenditure. The former possibility was eliminated as the result of a nitrogen balance study conducted in the laboratories of Robert Holland. The second possibility is consistent with activitywheel measurements of Lorens et al. (1970) and Grabowski and Smith (1974) and the restless, agitated behavior reported by Smith and Holland (1975).

\section{REFERENCES}

Anand, B. K. Nervous regulation of food intake. Physiological Review', 1965, 41, 677-708.

GRABowski. S. J., \& SMITh, M. O. Hyperactivity of accumbens lesioned rats and its effects on food intake. Unpublished B. A. Dissertation, Colgate University, 1974.

Harvey. J. A., \& Hunt, H. F. Effects of septal lesions on thirst in rats as indicated by water consumption and operant responding for water reward. Journal of Comparative and Physiological Psychology, 1965, 59, 49-56.

Hoebel, B. G. Feeding: Neural control of intake. Annual Review' of Physiology, 1971, 33, 533-568.

König. J. F..\& KLIPPEL, R. A. The rat brain: A stereotaxic atlas of the forebrain and lower parts of the brain stem. Baltimore: Williams \& Wilkins, 1963.

Lorens, S. A., Sorensen, J. P., \& Harvey, J. A. Lesions in the nuclei accumbens septi of the rat: Behavioral and neurochemical 
effects. Journal of Comparative and Physiological Psychology. 1970. 73. 284-290.

Lubar. J. F.. Schaefer. C. F.. \& Wells, D. G. The role of the septal area in the regulation of water intake and associated motivational behavior. Annals of the New York Academy of Sciences. 1969. 157. 875-893.
Smith, M. O..\& Holland. R. C. Effects of lesions of the nucleus accumbens on lactation and postpartum behavior. Physiological Psychology. 1975, 3, 331-336.

(Received for publication November 25. 1975; revision accepted April 7. 1976.) 